\title{
Correction: Early myeloma-related death in elderly patients: development of a clinical prognostic score and evaluation of response sustainability role
}

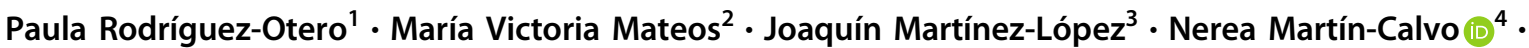 \\ Miguel-Teodoro Hernández ${ }^{5}$ Enrique M. Ocio ${ }^{2} \cdot$ Laura Rosiñol $^{6} \cdot$ Rafael Martínez $^{7} \cdot$ Ana-Isabel Teruel $^{8}$. \\ Norma C. Gutiérrez ${ }^{2} \cdot$ Joan Bargay $^{9}$. Enrique Bengoechea ${ }^{10}$. Yolanda González ${ }^{11}$ • Jaime Pérez de Oteyza ${ }^{12}$. \\ Mercedes Gironella ${ }^{13} \cdot$ Cristina Encinas $^{14} \cdot$ Jesús Martín $^{15} \cdot$ Carmen Cabrera $^{16} \cdot$ Luis Palomera $^{17} \cdot$ Felipe de Arriba $^{18}$. \\ María Teresa Cedena ${ }^{3} \cdot$ Bruno Paiva $^{1} \cdot$ Noemí Puig $^{2} \cdot$ Albert Oriol $^{19} \cdot$ Joan Bladé$^{6} \cdot$ Juan José Lahuerta ${ }^{4}$. \\ Jesús F. San Miguel ${ }^{1}$
}

Published online: 8 March 2019

(c) Springer Nature Limited 2019

\section{Correction to: Leukemia;}

https://doi.org/10.1038/s41375-018-0072-6;

published online 23 February 2018.

Following the publication of this article, the author notes that the following information was missed from the acknowledgments section:

These authors contributed equally: Paula Rodríguez-Otero, María Victoria Mateos

The original article can be found online at https://doi.org/10.1038/ s41375-018-0072-6.

Jesús F. San Miguel

sanmiguel@unav.es

1 Clínica Universidad de Navarra, CIMA, IDISNA, CIBERONC, Pamplona, Spain

2 Complejo Asistencial Universitario de Salamanca, Instituto de Investigación Biomédica de Salamanca, Salamanca, Spain

3 Hospital Universitario 12 de Octubre, Instituto de Investigación 12 de Octubre, CIBERONC, Madrid, Spain

4 Preventive Medicine and Public Health Department, University of Navarra, Pamplona, Spain

5 Hospital Universitario de Canarias, Santa Cruz de Tenerife, Spain

6 Hospital Clinic I Provincial, Institu d'Investigacions Biomèdiques August Pi i Sunyer (IDIBAPS), Barcelona, Spain

7 Hospital Clínico San Carlos, Madrid, Spain

8 Hospital Clínico de Valencia, Valencia, Spain

9 Hospital Son Llatzer, Palma de Mallorca, Spain
Acknowledgment of research support: This study was supported by the Cooperative Research Thematic Network grants RD12/0036/0058 and RD12/0036/0046 of the Red de Cancer (Cancer Network of Excellence); Instituto de Salud Carlos III, Spain, Instituto de Salud Carlos III/Subdirección General de Investigación Sanitaria part-financed by the European Regional Development Fund (FIS: PI12/ 01761; PI12/02311; PI13/01469; PI14/01867, G03/136; Sara Borrell: CD13/00340); Asociación Española Contra el Cáncer (GCB120981SAN) and FEDER.

The authors wish to apologise for any inconvenience caused.

10 Hospital de Donostia, San Sebastian, Spain

11 Institut d'Oncologia Dr. Josep Trueta, Girona, Spain

12 Hospital de Madrid Sanchinarro, Universidad CEU San Pablo, Madrid, Spain

13 Hospital Vall d'Hebron, Barcelona, Spain

14 Hospital General Universitario Gregorio Marañón, Instituto de Investigación Sanitaria Gregorio Marañón (IiSGM), Madrid, Spain

15 Hospital General Virgen del Rocío, Sevilla, Spain

16 Hospital San Pedro de Alcántara, Cáceres, Spain

17 Hospital Clínico Universitario Lozano Blesa, Zaragoza, Spain

18 Servicio de Hematología y Oncología Médica, Hospital Universitario Morales Meseguer, IMIB-Arrixaca, Universidad de Murcia, Murcia, Spain

19 Insitut Català d'Oncologia, Institut Josep Carreras, Hospital German Trias i Pujol, Badalona, Barcelona, Spain 